\title{
OCORRÊNCIA DE FATORES DE VIRULÊNCIA EM ESTIRPES DE ESCHERICHIA COLI ISOLADAS DE FEZES DE CÃES ERRANTES
}

\section{A.C.M.D.G . von Sydow, J.A. Coogan, A.M. M oreno, P.A. Melville, N .R. Benites}

Universidade de São Paulo, Faculdade de Medicina Veterinária e Zootecnia, Departamento de Medicina Veterinária PreventivaeSaúdeA nimal, A v. Prof. Dr. Orlando Marques dePaiva, 87, CEP 05508-000, São Paulo, SP, Brasil. E-mail: aguercio@usp.br

RESUMO

Escherichia coli pode causar doenças em humanos e animais, tais como infecções do trato urinário, septicemia, meningites e gastroenterites. Cães assintomáticos podem participar da cadeia epidemiológica como reservatório deE. coli patogênica ao homem. Este estudo objetivou avaliar a ocorrência de E. coli patogênica ao homem em fezes de cães errantes sem sintomas de colibacilose e assim averiguar a participação do cão como fonte de infecção de colibacilose humana. Foram coletadas 220 amostras defezes deanimais capturados por diferentes Centros de Controle de Zoonoses do Estado de São Paulo. As amostras foram submetidas a exames microbiológi cos sendo isoladas 196(89,09\%) estirpes deE. coli, cujosgenes defatores devirulência foram detectados por PCR. Cento evinte etrês $(62,75 \%)$ estirpes apresentaram um ou mais dos fatores de virulência estudados. Dezesseis (8,16\%) foram positivas para afa, 54 (27,55\%) para sfa, $38(19,38 \%)$ para pap, $66(33,67 \%)$ para aer , $31(15,81 \%)$ para cnf, $13(6,63 \%)$ para hly, $1(0,51 \%)$ para VT2enenhumadas linhagensfoi positiva paraLT, STaeSTb. Cães errantes aparentementesadios podem participar da cadeia epidemiológica como reservatórios de E. coli uropatogênica ao homem, pois os genes encontrados com maior freqüência estão presentes em infecções extraintestinais urinárias.

PALAVRAS-CHAVE: Escherichia coli, fezes, cães, fatores de virulência, PCR.

\section{ABSTRACT}

OCCURENCE OF VIRULENCE FACTORSIN LINEAGESOF ESCHERICHIA COLI ISOLATED FROM FAECESOF STRAY DOGS. Escherichia coli may causediseases in humans and animals, such asurinary infections, septicemia, meningitisand gastroenteritis. Asymptomaticdogscan participate in theepidemiol ogic chain as a reservoir of human pathogenicE. coli. Theobjective of the present study was to evaluatetheoccurrence of pathogeni cE. coli in humans, on faeces of stray dogswithout colibacillosissymptoms, and thusverify theparticipation of dogsasasourceof human colibacillosis infection. Two hundred and twenty samples of faecesfrom animals captured by differentZoonotic Control Centers in São Paulo state, Brazil, were collected. These faeces, when submitted to microbiological exams, resulted in theisolation of 196 (80.09\%) strains of E. coli, whosevirulence genesweredetected by PCR. A t leastoneof thevirulencefactorsstudied waspresent in 123(62.75\%) strains. Sixteen (8.16\%) were afa positive, 54 (27.55\%) were positive to sta, 38 (19.38\%) to pap, 66 (33.67\%) to aer, 31 (15.81\%) to cnf, $13(6.63 \%)$ to hly, $1(0.51 \%)$ to VT2, and none of the strains were positive for LT, STa or STb. Stray dogs which were apparently healthy could be participating in theepidemiologic chain as anE. coli reservoir uropathogenicto man, as thegenesfound in ahigher frequency are present in extraintestinal infections, or more specifically, urinary infections.

KEY WORDS: Escherichia coli, faeces, dogs, virulence factors, PCR.

\section{INTRODUÇÃO}

E scherichia coli éconsiderada habitantenatural do trato intestinal de animais edo homem, mas também aparece como uma importante causa de diarréias, infecções urinárias, mastites, septicemias, meningi- tes. Temsido responsável por causar diarréia seguida demorteem crianças depaíses em desenvolvimento, assimcomocolitehemorrágica(HC), síndromeurêmica hemolítica (HUS) em crianças e adultos em países desenvolvidos. Veículos deinfecçãotêmsidoalimentos de origem animal, água ealimentos mal lavados 
como frutas, verduras e raízes. (Drolet et al., 1994; Sussman, 1997; Trabulsi; Campos, 1999; Guth et al., 2002; Y ATSUYANAGI et al., 2002).

A habilidade de E. coli em causar doença em humanos é devida à presença de vários fatores de virulência localizados em genes plasmidiais e/ ou cromossomais DonnenberG; WhitTam, 2001). A patogenicidadedomicrorganismo estárelacionadaà forma como a bactéria seliga à célula do hospedeiro, à produção de toxinas e a sua invasão (SEARS; KAPER, 1996; FARMER III, 1999).

Existem relatos da ocorrência de colibacilose em cães e gatos, além de outros animais, tendo sido relatadas infecções urinárias, prostatites, vaginitese piometra, além desepticemiaseendotoxemias(BEUTIN 1999).

O grupo das E. coli uropatogênicas (UPEC) está associadoadoençasno tratourinário tanto em humanoscomo emanimais. As adesinasmais importantes nas infecções urinárias são ospili. AsfímbriasS(sfa) estimulamaprodução del nterleucina-6pelascélulas doepitéliorenal napresençadeUTI (infecçãodo trato urinário) (SAYLERS; WHITT, 1994). As fímbrias tipo 1 participam na colonização da bexiga etrato urinário inferior. A adesina das estirpes que infectam os rins éafímbriaP, queestáassociadaàspielonefrites (CHEN et al., 2003).

Hemolisinas são citotoxinas (HlyA) que conduzemaumaliseosmóticaprincipalmentedeeritrócitos (Trabulsi; Campos, 1999; Ribeiro, 2001). E. coli uropatogênicas podem apresentar a habilidade de adquirir ferro, utilizando sideróforos, exoproteínas codificadas pelo geneaer plasmidiais (SAYLERS;W HITT, 1994). A aerobactina, sideróforo bacteriano, tem sido associada a estirpes deE. coli quecausam pielonefrite e cistite em humanos (Johnson et al ., 1988).

O fator necrotizante citotóxico (NTEC), está associado com infecções extraintestinais (septicemia e infecção do trato urinário) e enterites no homem e animais (SEARS; KAPER, 1996; RIBEIRo, 2001).

Sete fatores de virulência (FVs), incluindo pilus tipo 1 (pili), pilus associado com piel onefrite (pap), fímbria S (sfa), adesina afimbrial I (afa I), hemol isina (hly), aerobactina (aer) efator citotóxico necrotizante (cnf) desempenham um importante papel no desenvolvimento de UTI humanas. Linhagens de E. coli isoladas de cães e gatos com UTI são similares às estirpes deE. coli isoladas de UTI humana. Pili foi o fator devirulênciamaiscomumenteencontrado entre as linhagens UPEC isoladas de cães e gatos e de humanos. Além disso, estafímbriafoi freqüentemente observada em estirpes deE. coli isoladas de fezes de cães egatossadios. O genecodificador dafímbriaP foi encontrado com maior freqüência em fezes de cães saudáveis (YuRI et al., 1998; Kurazono et al., 2003). Escherichia coli isolada de fezes de cães, comumente exi bem características típicas de ExPEC humana (E. coli extraintestinal), podendo ser relacionados com isolados clínicos de pacientes humanos com cistite, pielonefrite, bacteremia ou meningite (JoHnson et al., 2001).

Cãestêmsido apontados como potenciais reservatórios de linhagens de E. coli que causam UTI em humanos, pelo fato destes microrganismos apresentarem proximidadefilogenética, desorogrupos ecertos fatores de virulência associados com $E$. coli extraintestinal isolados de fezes e urina caninos, sugerindo a ocorrência de transmissão de $\mathrm{E}$. coli patogênica entrecães ehumanos (SANNEs et al ., 2004).

Cães e gatos compõem um grupo de animais de grandeproximidadeao homem. Comoconseqüência, a possibilidade de transmissão de microrganismos entre estas espécies de hospedeiros é extremamente elevada (BEutin, 1999).

O presente estudo teve como objetivo verificar a ocorrência deE. coli nas fezes de cães errantes, com ausência de sintomas de colibacilose, avaliando a possível presença de fatores de virulência nessas estirpesqueapresentam similares às observadas nas linhagens isoladas de infecções em humanos.

\section{MATERIAISE MÉTODOS}

\section{Coleta das amostras}

A coleta do material foi realizada nas dependências dos Centros de Controle de Zoonoses (CCZs) pertencentes aos Municípios de Guarulhos, Cotia e Barueri, Estado deSão Paulo. Foram colhidas amostras de fezes de 220 cães recolhidos pelos CCZ das cidades referidas. A escol ha dos cães foi realizada independentemente de raça, porte, idade ou sexo, levando-seem consi deração o estado geral eaconsistência das fezes que deveriam indicar ausência de diarréia.

A coletadeamostras defezesfoi feitanomomento em que os animais apresentavam sinais clínicos de anestesia, antes deserem eutanasiados. Tal coletafoi realizada com o auxílio de suabe estéril introduzido profundamente ao intestino (porção retal), após os animais terem sido anestesiados.

\section{Exames microbiológicosdeamostrasdefezesdecães}

Ossuabes foramencaminhadosaolaboratório em condição derefrigeração, condicionados em geladeiraportátil deisopor com gelo reciclável. No laboratório, foram submetidos aos exames microbiológicos queconsistiram inicial menteno cultivo dos mesmos em caldo BHI (brain and heart infusion broth), ágar sangue de carneiro (5\%) (blood agar base) e ágar 
MacConkey (MacConkey agar) com incubação em aerobiose, a $37^{\circ} \mathrm{C}$ com leituras a 24-96h. As amostras também foram cultivadas em ágar Sabourauddextrose (Sabouraud dextrose agar) com incubação em aerobiose, mantidas em temperatura ambiente, por um período mínimo de 7 dias. As amostras incubadas em cal do BHI foram posteriormentesemeadas em ágar sangue de carneiro (5\%) eágar M acConkey, sendo a incubação destas similar à descrita anteriomente para o cultivo inicial.

Os microrganismos isolados foram identificados de acordo com LenNETTE et al. (1985) e classificados segundo Kreeger-Van-RIg (1984), KRIEG; H olt (1994), FARMER III et al. (1999).

Pesquisa dos genes codificadores dos fatores de virulência deE. coli.

Como controles positivospara os diferentesgenes pesquisados foi empregada amostra de E. coli enterotoxigênica e verotoxigênica pertencentes à coleção deculturas do Laboratório de SanidadeSuínaVPS- FMVZ-USP. Estas amostras foram previamente caracterizadas através de provas fenoti picas quanto à produção dastoxinasSTa, STb, LT eVT. Ascepasde
E. coli uropatogênicasforam cedidaspeloLaboratório deOrnitopatologia-VPT/ FMVZ-USP sendo previamente caracterizadas quanto à presença destes fatores por métodos fenotípicos.

\section{Extração do DNA bacteriano}

Para extração do DNA genômico as amostras foram cultivadas em infusão cérebro coração (BHI), a $370 \mathrm{C}$ por $18 \mathrm{~h}$. Uma al íquota de $200 \mu \mathrm{L}$ da cultura foi submetidaà extração deDN $A$ através do método baseado na utilização de isothiocianato de guanidina descrito por Boom et al. (1990). A samostras de DNA foram mantidas a $-20^{\circ} \mathrm{C}$ até sua utilização na PCR.

\section{Reação em cadeia da polimerase}

Osoligonucleotídeos iniciadores (primers)específicos para os genes codificadores dos fatores de virulência estudados foram sintetizados pela Life Technologies (Miami) e são apresentados no Tabela 1. Foram realizadas duas combinações de primers para pesquisa dos genes. O primeiro multiplex consistiu da pesquisa dos genes para STa, STb. LT eVT1 eVT2, o segundo agrupou todos os fatores relacionados a amostras uropatogênicas.

Tabela 1 - "Primers" utilizados na PCR para amplificar fragmentos de diferentes genes para enterotoxinas (LT, STa e STb) e vero citotoxinas (VT1, VT2 e VT2e) e de diferentes genes para pap, sfa, afa, hly, aer e cnf.

\begin{tabular}{|c|c|c|c|c|}
\hline $\begin{array}{l}\text { Genes } \\
\text { codificadores } \\
\text { das toxinas }\end{array}$ & $\begin{array}{l}\text { Nome do } \\
\text { primer }\end{array}$ & Seqüência do oligonucl eotídeo $\left(5^{\prime} \rightarrow 3^{\prime}\right)$ & $\begin{array}{l}\text { Tamanho do } \\
\text { produto } \\
\text { amplificado }\end{array}$ & Referência \\
\hline \multirow[t]{2}{*}{ LT } & LTA-1 & GGCGACAGATTATACCGTGC & $696 \mathrm{bp}$ & Schultsz et al. (1994) \\
\hline & LTA-2 & CCGAATTCTGTTATATATGTC & & \\
\hline \multirow[t]{2}{*}{ STa } & STI-1 & TTAATAGCACCCGGTACAAGCAGG & $147 \mathrm{bp}$ & OLsvik et al. (1993) \\
\hline & STI-2 & СTTGACTCTTCAAAAGAGAAAATTAC & & \\
\hline \multirow[t]{2}{*}{ STb } & STb-1 & ATCGCATTTCTTCTTGCATC & 172 bp & BLAnco et al. (1997) \\
\hline & STb-2 & GGGCGCCAAAGCATGCTCC & & \\
\hline \multirow[t]{2}{*}{ VT1 } & VT1-A & GAAGAGTCCGTGGGATTACG & $130 \mathrm{bp}$ & Pollard et al. (1990) \\
\hline & VT1-B & AGCGATGCAGCTATTAATAA & & \\
\hline \multirow[t]{2}{*}{ VT2 hb² } & VT2-3 & CCGTCAGGACTGTCTGAAAC & 726 bp & WoodWARD et al. (1992) \\
\hline & VT2-5 & GAGTCTGACAGGCAACTGTC & & \\
\hline \multirow[t]{2}{*}{ Pap } & pap-1 & GCAACAGCAACGCTGGTTGCATCAT & 336 bp & Yамамото et al. (1995) \\
\hline & pap-2 & AGAGAGAGCCACTCTTATACGGACA & & \\
\hline \multirow[t]{2}{*}{ Hly } & hly-1 & AACAAGGATAAGCACTGTTCTGGCT & $1.177 \mathrm{bp}$ & Yамамото et al. (1995) \\
\hline & hly-2 & ACCATATAAGCGGTCATTCCCGTCA & & \\
\hline \multirow[t]{2}{*}{ A er } & aer-1 & TACCGGATTGTCATATGCAGACCGT & 602 bp & Yамамото et al . (1995) \\
\hline & aer-2 & AATATCTTCCTCCAGTCCGGAGAAG & & \\
\hline \multirow[t]{2}{*}{$\mathrm{Cnf}$} & cnf1 & AAGATGGAGTTTCCTATGCAGGAG & $498 \mathrm{bp}$ & Yамамото et al. (1995) \\
\hline & cnf2 & САTTCAGAGTCCTGCССTCATTATT & & \\
\hline \multirow[t]{2}{*}{ Sfa } & sfa-1 & CTCCGGAGAACTGGGTGCATCTTAC & $410 \mathrm{bp}$ & ҮАмАMOTO et al. (1995) \\
\hline & sfa-2 & CGGAGGAGTAATTACAAACCTGGCA & & \\
\hline \multirow[t]{2}{*}{ A fã } & afa-1 & GCTGGGCAGCAAACTGATAACCTC & 750 bp & Yамамото et al. (1995) \\
\hline & afa-2 & CATCAAGCTGTTTGTTCGTCCGCCG & & \\
\hline
\end{tabular}


A solução deamplificação padrão dePCR consistiu de $10 \mathrm{mM}$ Tris- $\mathrm{HCl}(\mathrm{pH} 8,3), 50 \mathrm{mM} \mathrm{KCl}, 1,5 \mathrm{mM}$ $\mathrm{MgCl}_{2}$, gelatina $0,001 \%(\mathrm{w} / \mathrm{v}), 200 \mu \mathrm{M}$ decadaumdos desoxinucleosídeos trifosfatos, 20 pmoles da cada primer e $0,2 \mu \mathrm{L}$ de Taq DNA polimerase, $5 \mu \mathrm{L}$ da amostra deDNA eágua atéo volume final de $50 \mu \mathrm{L}$.

O programa utilizado para todas as reações consistiu de 1 ciclo a $95 \circ \mathrm{C}$ por $5 \mathrm{~min}, 35$ ciclos a $95 \circ \mathrm{C}$ por $1 \mathrm{~min}, 55^{\circ} \mathrm{C}$ por $1 \mathrm{~min}, 72^{\circ} \mathrm{C}$ porl min eum ciclo final de $72^{\circ} \mathrm{C}$ por $5 \mathrm{~min}$ (Termociclador).

A cadaamplificação realizada, foi adicionado um controle positivo contendo o DNA daE. coli positiva para o gene pesquisado e um controle negativo contendo os reagentes sem DNA.

\section{D etecção do produto amplificado}

Os produtos de PCR (10 $\mu \mathrm{L})$ foramseparadospor eletroforese em gel de agarose $2 \%$, a 4 volts/ cm. A pós a corrida, o gel foi corado com brometo de etídio ( $10 \mu \mathrm{g} / \mathrm{mL}$ ) efotografado sobluzultravioleta. Foi utilizado o marcador molecular 100 bp DNA ladder.

\section{Estatística}

A sanál isesestatísticasforamreal izadasutilizando-se o teste de Fisher, empregando-se o software GraphPad Instat 3.

\section{RESULTADOS}

Examesmicrobiológicosdeamostrasdefezesde cães errantes

Em 196 (89,09\%) das 220 amostras defezes, foram isoladas $120(54,55 \%)$ estirpes deE. coli emculturapura e76 (34,54\%) estirpes em associação com outrosagentes, dentre outros microrganismos (Tabela 2). A freqüência deisolamentos deE. coli (89,09\%ou 196amostras), considerando-seas amostras nas quaiso agente foi isolado em cultura pura e também em associação comoutrosmicrorganismos, foi estatisticamentemais significante $(P<0,05)$ do que as freqüências de isolamentos de todos os outros microrganismos.

Tabela2- Freqüência deocorrência demi crorganismos (bactérias efungos) em cultura pura ou emassociações, isolados a partir de 220 suabes retais de cães errantes.

\begin{tabular}{|c|c|c|}
\hline Microrganismos isolados & $\mathrm{N}$ & $\%$ \\
\hline E. coli & 120 & $54,55^{*}$ \\
\hline E. coli / Proteus mirabilis & 23 & 10,45 \\
\hline E. coli / K lebsiella pneumoniae & 8 & 3,64 \\
\hline E. coli / Edwarsiela tarda & 6 & 2,73 \\
\hline E. coli / Proteus mirabilis / Klebsiella pneumoniae & 5 & 2,27 \\
\hline E. coli / Klebsiella pneumoniae / Staphylococcus sp. & 4 & 1,82 \\
\hline E. coli / Proteus mirabilis / Staphylococcus sp. & 4 & 1,82 \\
\hline E. coli / Staphylococcus sp. & 4 & 1,82 \\
\hline E. coli / K lebsiella pneumoniae / Streptococcus sp. & 2 & 0,91 \\
\hline E. coli / Proteus mirabilis / Candida albicans & 2 & 0,91 \\
\hline E. coli / Proteus mirabilis / Edwarsiella tarda & 2 & 0,91 \\
\hline E. coli / Pseudomonas sp. & 2 & 0,91 \\
\hline E. coli / C andida al bicans & 1 & 0,45 \\
\hline E. coli / C andida albicans / Klebsiella oxytoca & 1 & 0,45 \\
\hline E. coli / Klebsiella pneumoniae / Candida albicans & 1 & 0,45 \\
\hline E. coli / Klebsiella pneumoniae / Staphylococcus sp. / Streptococcus sp. & 1 & 0,45 \\
\hline E. coli / Proteus mirabilis / K lebsiella pneumoniae / Salmonella sp. & 1 & 0,45 \\
\hline E. coli / Proteus mirabilis / Streptococcus sp. & 1 & 0,45 \\
\hline E. coli / Proteus vulgaris / Citrobacter amanolaticus & 1 & 0,45 \\
\hline E. coli / Providencia sp. & 1 & 0,45 \\
\hline E. coli /K lebsiella oxytoca & 1 & 0,45 \\
\hline E. coli / Proteus vulgaris & 2 & 0,91 \\
\hline E. coli / Staphylococcus sp. / Proteus vulgaris & 1 & 0,45 \\
\hline E. coli / Streptococcus sp. & 1 & 0,45 \\
\hline E. coli / Streptococcus sp. / Citrobacter amalonaticus & 1 & 0,45 \\
\hline E. coli / outros & 76 & 34,54 \\
\hline
\end{tabular}

Continua 
Tabela 2 - Continuação.

\begin{tabular}{lll}
\hline Microrganismos isolados & N & $\%$ \\
\hline Proteus mirabilis & 6 & 2,73 \\
Proteus mirabilis / K lebsiella pneumoniae & 2 & 0,91 \\
Salmonella sp. & 2 & 0,91 \\
Klebsiella pneumoniae / Providencia sp. & 1 & 0,45 \\
Proteus mirabilis / Edwarsiella tarda & 1 & 0,45 \\
Proteus mirabilis / Klebsiella pneumoniae / Staphylococcus sp. & 1 & 0,45 \\
Proteus mirabilis / Salmonella sp. / Streptococcus sp. & 1 & 0,45 \\
\hline
\end{tabular}

*Verificou-se diferença estatisticamente $(P<0,05)$ significante entre a ocorrência de Escherichia coli e os outros microrganismos isolados.

Tabela3-Freqüência deocorrênciadefatores devirulência em estirpes de E. coli em fezes de cães sem diarréia.

\begin{tabular}{llr}
\hline Fatores de Virulência & N & \multicolumn{1}{c}{$\%$} \\
\hline afa & 16 & 8,16 \\
sfa & 54 & 27,55 \\
pap & 38 & 19,38 \\
aer & 66 & 33,67 \\
cnf & 31 & 15,81 \\
hly & 13 & 6,63 \\
VT2 & 1 & 0,51 \\
\hline
\end{tabular}

Legenda: pili associados com pielonefrite (pap); aerobactina (aer); fator necrotizante citotóxico (nf); fímbria S (sfa); adesina afimbrial I (afa); hemolisina (hly); verotoxina 2 (VT2).

\section{Pesquisa dos genes codificadores dos fatores de virulência de E. coli utilizando PCR}

Deum total de 196 estirpes deE. coli isoladas, 123 $(62,75 \%)$ apresentaram um ou mais dos fatores de virulência estudados. Destas estirpes, $16(8,16 \%)$ foram positivas paraafa, $54(27,55 \%)$ parasfa, $38(19,38 \%)$ para pap, 66 (33,67\%) paraaer, 31 (15,81\%) paracnf, 13 $(6,63 \%)$ parahly, $1(0,51 \%)$ para VT2 enenhuma das linhagens foi positiva para LT, STa eSTb (Tabela 3). Do total de linhagens estudadas, 61 (49,59\%) apresentou mais de um tipo de fator de virulência.

Os fatores de virulência aer e sfa apresentaram freqüência de ocorrência significantemente maior $(P$ $<0,05)$ quando comparados comafa, cnf, hly, LT, STa, STb eVT. Por sua vez, pap apresentou freqüência de ocorrênciasignificantementemaior $(P<0,05)$ queafa, hly, LT, STa, STb eVT.

\section{DISCUSSÃO}

O isolamento deestirpes deE .coli emumafreqüência de $89,09 \%$, em cultura pura ou associação com outros microrganismos, demonstrou a el evada ocorrência destemicrorganismo emfezes decães hígidos, a qual é estatisticamente $(P<0,05)$ maior do quedos outros agentes isolados. Segundo SANCAK et al. (2004), E. coli é um dos principais componentes da flora intestinal dos seres humanos e dos animais.

De acordo com Beutin (1999), estirpes de E. coli uropatogênicas isoladas da flora fecal edeinfecções extra-intestinais decãesforamsimilaresàslinhagens uropatogênicas isoladas de humanos quanto aos seus atributos de virulência. Segundo este pesquisador, os cães devem apresentar um papel importante como reservatórios destemicrorganismo para outros animais e para o homem, pois estudos indicam a transmissão de linhagens uropatogênicas fecais entre humanos e cães. Entretanto, pouco se conhece sobreo modo detransmissão dasE. coli uropatogênicas entre os diferentes hospedeiros mamíferos.

No presente estudo foram identificados fatores de virulência em $62,75 \%$ das linhagens avaliadas. Os fatores de virulência detectados com maior freqüência foram os genes aer $(33,67 \%)$, sfa $(27,55 \%)$ e pap (19,38\%), os quais estão freqüentementeassociados a casos de infecções urinárias e/ ou genitais humanas.

KURAZONo et al. (2003) apontaram a presença de genes pap, sfa, afa, hly, aer e cnf em estirpes de E. coli isoladas defezesnormaiseurinadecães. Por suavez, FÉrIA et al. (2000) detectaram diferentes fatores de virulência em estirpes isoladas de infecção do trato urinário de humanos, cães e gatos, sendo que aerobactina (aer) apresentou a maior freqüência entre os gatos, hemolisina (hly) entre os humanos e pili associados a piel onefrite ( pap) entre os cães, sugerindo uma adaptação deE. coli uropatogênica aosreceptores celulares de cada hospedeiro. No presente estudo por sua vez, os fatores devirulência encontrados com maior freqüência foramaer, sfa epap, embora também tenham sido verificados, em freqüência inferior, a hemolisina (hly) efator citotóxico necrotizante (cnf). 
YuRi et al . (1998) verificaramapresença defatores de virulência, incluindo pili associados com pielonefrite (pap), fímbria S (sfa), adesina afimbrial (afa I), alfa hemolisina (hly), fator citotóxico necrotizante 1 ( $\mathrm{cnf} 1$ ) e aerobactina (aer) em estirpes isoladas de urina de cães e gatos com UTI efezes de animaishígidos. Estesfatores devirulênciatambém são encontrados em estirpes isoladas de humanos. As linhagens isol adas de humanos e cães com UTI possuem mai or quantidadedefatores devirulência do que aquelas encontradas em humanos e cães saudáveis.

Tendo em vista queE scherichia coli éum dos mais freqüentes agentes causais de infecções do trato urinário de humanos, tanto quanto de cães egatos, é importanteo seu conhecimento, particularmentequando seconsidera o convívio próximo entreestas espé cies. N opresenteestudo, osfatores devirulênciamais encontrados foram uropatogênicos (aer, sfa e pap) concordando com as observações deY uRI et al. (1998), embora os animais estudados fossem cães errantes e não apresentassem sinais clínicos de UTI. Low et al . (1988) estudaram E. coli isoladas do reto e do trato urinário de cães com infecção urinária e concluíram que os isolados de urina poderiam ser de origem intestinal. Emmulheres, amicrobiotafecal éreconhecidacomo reservatório demicrorganismos potencialmente causadores de infecções bacterianas do trato urinário (WADAS, 1996).

Estudo realizado por JoHnson et al. (2001) apontou semelhanças entre amostras de ExPEC $E$. coli Patogênica Extraintestinal) humana e canina com relação aos fatores de virulência, baseado no polimorfismo genético, mas não comprovou a transmissão cruzada entre as espécies ou excluiu a possibilidade de humanos ecaninos adquirirem o mesmo tipo deE. coli de origem externa comum.

O cão errante poderia estar contami nando o meio ambiente e expondo o ser humano a enterites por $\mathrm{E}$. coli eatémesmo outrasmanifestações decolibacilose, através do risco de contaminação de mananciais ou pel o contato direto quepessoas poderiam vir ater com estes animais os quais, desta forma, atuariam como fontes de infecção na ocorrência de colibacilose humana (Blanco et al., 1997; KrUth, 1998; Farmer III, 1999).

Oscãeserrantes aparentementesadios, semsintomas de colibacilose, poderiam estar participando da cadeia epidemiológica como reservatórios deE. coli (microrganismo isolado com maior freqüência das fezes destes animais) de natureza uropatogênica ao homem, pois os genes detectados em mai or frequência foramaer, sfa e pap, presentes em linhagens associadas a infecções extraintestinais, mais especificamente infecções urinárias. Deve-se proceder à realização de mais estudos que visem à identificação do modo como pode ocorrer a transmissão deste agente entre cães ehumanos, o que possi bilitaria o desenvolvimento de mecanismos de prevenção da doença.

\section{REFERÊNCIAS}

BEUtIN, L. Escherichia coli as a pathogen in dogs and cats. Veterinary Research, v.30, p.285-298, 1999.

Blanco, M.; Blanco, J.E.; Gonzalez, E.A.; Mora, A.; Jansen, W.; Gomes, T.A.T.; Zerbini, F.; Yano, T.; Pestana de CASTRO, L.; BLANCO, J. Genes coding for enterotixins and verotoxins in porcine Escherichia coli strains belonging to different $\mathrm{O}: \mathrm{K}: \mathrm{H}$ serotypes: relationship with toxic phenotypes. Journal of Clinical M icrobiology, v.35, p.2958-2963, 1997.

Boom, R.; Sol, C.J.A.; Salimans, M.M.M.; Ansen, C.L.; Werthein-Van Dilen, P.M.E.; N oordaa Van DerJ.Rapid and simple method for purification of nucleic acids. Journal of Clinical M icrobiology, v.28, p.495-503, 1990.

Chen, Y. M. M.; Wright, P. J.; LeE, C. S.; Browning, G. L. Urophatogenic virulence factors in isolates of Escherichia coli from clinical cases of caninepyometra and feces of healthy bitches. V eterinary M icrobiology, v.94, p.57-69, 2003.

Donnenberg, M.S.; Whittam, T.S. Pathogenesis and evolution of virulence in enteropathogenic and enterohemorrhagic Escherichia coli. TheJ ournal ofC linical Investigation, v.107, p.539-548, 2001.

Drolet, R.; Fairbrother, J.M.; H arel, J.; Hélie, P. Attaching and effacing and enterotoxigenic Escherichia coli associated with enteric colibacillosis in the dog. Canadian Journal Veterinary Research, v.58, p.87-92, 1994.

FARMER III, J.J. Enterobacteriaceae: Introduction and Identification. In: Murray,P.R.;B ARon, E.J.;P Faller, M.; Tenover, F.C.; Yolken, R.H. (Eds.). M anual of clinical microbiology. 7.ed. Washington: American Society for Microbiology, 1999. cap.27, p.442-455.

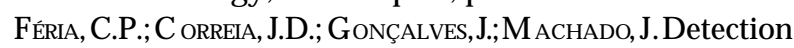
of virulence factors in Uropathogenic Escherichia coli isol ated from humans, dogs and cats in Portugal. A dvances in Experimental M edicineand Biology, v.485, p. 305-308, 2000.

Guth, B.E.C.; Ramos, S.R.T.S.; Cerqueira, A.M.F.; Andrade, J.R.C.; Gomes, T.A.T. Phenotypic and genotypic characteristics of shiga toxin-producing Escherichia coli strainsisolated from children in São Paulo, Brazil. $M$ embro do Instituto 0 swaldo Cruz, v.97, n.8, p.10851089, 2002.

Johnson, J.R.; Moseley, S.L.; Roberts, P.L.; Samm, W.E. A erobactin and other virulence factor genes among strains of Escherichia coli causing urosepsis: Association with pacients characteristics. Infection and Immunity, v.56, p.405- 412, 1988.

Johnson, J.; Stell, A.L.; Delavari, P. Canine feces as a reservoir of extraintestinal pathogenic Escherichia coli. Infection and Immunity, v.69, n.3, p.1306-1314, 2001.

KeGer-VAN-RIG, N.J.N. The yeats a taxonomic study. 3.ed. A msterdan: Elsevier Science Publisher, 1984. 
KRIEG,N.R.;H oLt, J.C.Bergey's manual of sistematicbacteriology. 9.ed. Baltimore: Williams e Wilkins, 1994. 894p.

$\mathrm{K}_{\text {RUTH }}, \mathrm{S}$. A..Gramnegativebacterial infections. In:GreEnE, C.E. (Ed.). Infections diseases of dog and cat. 2.ed. Philadel phia: W.B. Saunders, 1998. p.217-222.

Kurazono, H.; Nakano, M.; Yamamoto, S.; Ogawa, O.; Yurl, K.; Nakata, K.; Kimura, M.; Makino, S.; Nair, G.B. Distribution of the usp gene in uropathogenic Escherichia coli isolated from companion animals and correlation with serotypes and size-variations of the pathogenicity island. M icrobiololy and I mmunology, v.47, p.797-802, 2003.

Lennete, E.H.; Hansler Junior, W.J.; Shadomy, H.J. Manual of clinical microbiology. 4.ed.Washington:A merican Society for Microbiology Press, 1985. 1149p.

Low, D.A.;BraAten, B.A.; Ling, G.V.; Johnson , D.L.; Rugy, A.L. Isolation and comparasion of Escherichia coli strains from canine and human patients with urinary tract infections. Infection Immunology, v.56, p. 2601-2609, 1988.

Olsvik, O.J. Wahlberg, B. Petterson, M. Uhlen, T. Popovic.I. K. Wachsmuth, And P.I. Felds. Use of automated sequecing of polimerase chain reation-generated amplions to identify three types of cholera toxin subunit B in Vibrio cholerae O1 strains. Journal of Clinical M icrobiology, v.31, n.1, p.22-25, 1993.

Pollard, D.R.; Johnson, H.L.; Lior, H.; Tyler, S.D.; Rozee, K.R. Rapid and specific detection of verotoxin genes in Escherichia coli by the polymerase chain reaction. Journal of Clinical M icrobiology, v.28, p.540545, 1990.

RıbeIRo, M.G. Fatores devirulência em linhagens deE.coli isoladas demastitebovina clínica esub-clínica. 2001. 70f. Tese(Doutorado em Epidemiologia Experimental aplicada as Zoonoses e Medicina Veterinária Preventiva e Saúde A nimal) - Faculdade de Medicina Veterinária e Zootecnia, UniversidadedeSãoPaulo, São Paulo, 2001.

Sancak, A.A.; Rutgers, H.C.; Hart, C.A.; Batt, R.M. Prevalence oh enteropathic Escherichia coli in dogs with acute and chronic diarrhoea. The V eterinary Record, v.154, p.101-106, 2004.

SAnnes, M.R.; Kuskowskı, M.A.;J ohnson , J.R..A ntimi crobial resistance of Escherichia coli strains isolated from urine of women with cystitis or pyelonephritis and feces of dogs and healthy humans. Journal of the A merican Veterinary M edical Association, v.225, n.3, p.368-373, 2004.

SAYLERS, A.A; WhITT, D.D. Escherichia coli Gastrointestinal Infections. In . Bacterial Pathogenesis: a molecular approach. Washington, DC: American Society for Microbiology, 1994. chap.16., p.190- 212.
SAylers, A.A; WhITt, D.D. Escherichia coli Urinary Tract Infections. In Bacterial Pathogenesis: a molecular approach. Washington, DC: American Society for Microbiology, 1994. chap. 17., p.205- 212.

Schultsz C.;Pool,G.J.;V an Ketel,R.;D e Wever, B.;SPeelman , P.; DANKERT, J. Detection of enterotoxigenic E. coli in stool samples by using nonradiatioactively labeled oligonucleotide DNA probes and PCR. Journal of Clinical M icrobiology, v.32, p.2393-2397, 1994.

SeARS, C.L.;K APER, J.B. Entericbacterial toxins:mechanisms of action and linkage to intestinal secretion. M icrobiology Reviews, v.60, p.167-215, 1996.

Stone, G.G., Oberst, R.D., Hays, M.P., Vey, S.Mc, Chengappa, M.M. Detection of salmonella serovars from clinical samples by enrichment broth cultivation - PCR procedure. Journal ofM icrobiology,v.32,p.1742-1749, 1994.

Sussman, M. (Ed.). Escherichia coli: mechanisms of virulence. Cambridge: CambridgeU niversity Press, 1997.639p.

TrabulSI, L.R.; Campos, L.C. Escherichia. In: Trabulsi, L.S; SouzA, C.P. (Eds.). M icrobiologia. São Paulo: Atheneu, 1999. Cap. 28. p.215-228.

WAdAS, B.;KüHn, I.;LAGERSTEDT,A.S.;j OnSSON , P. Biochemical phenotypes of Escherichia coli in dogs: comparison of isolatesisolated from bitchessuffering frompyometra and urinary infection with isolates from faeces of heal thy dogs. V eterinary M icrobiology, v.52, p.293-300, 1996.

WoOdWARD, M.J.;CARROLL,P.J.;WRAY, C. Detection of enteroand verocyto-toxin genes in Escherichia coli from diarrhoeal disease in animals using the polymerase chain reaction. Veterinary $M$ icrobiology, v.31, p.251261, 1992.

YATSUYANAGI, J.; SAITO, S.; I to, I .. A caseof hemolytic uremic sindrome associated shiga toxin 2 -producing Escherichia coli $\mathrm{O} 121$ infection caused by drinking water contaminated with bovinefeces.J apanesej ournal Infection Disease, v.55, p.174-176, 2002.

Yurl , K.; N akata, K.; Katae, H.; Y amamoto, S.; H asegawa,A. Distribution of uropathogenic virulence factors among Escherichia coli strains isolated from dogsand cats. J ournal of V eterinary M edicine Science, v.60, n.3, p.287-290, 1998.

Yuri, K.; N akata, K.; K atae, H.;T Tokamoto, T.; H asegawa, A. Serotypes and virulence factors of Escherichia coli strainsisolated from dogsand cats.J ournal of $V$ eterinary M edicine Science, v.61, n.1, p.37-40, 1998.

Recebido em 24/ 5/ 06

A ceito em 29/ 10/ 06 